\title{
Preface to Special Issue on Carbon in Catalysis
}

"Carbon" is a magic word in the scientific community that means more than just the sixth chemical element with the symbol C, which is found in all organic molecules and is present in all forms of life. The best known allotropes of carbon graphite, diamond, and activated carbon and carbon black have become a huge industry. Since the beginning of the 1990s, the discovery of fullerenes, carbon nanotubes, graphene, and nanocarbons such as nanographite, nanodiamond, nanohorns, and nanospheres has attracted general attention and tempted scientists all over the world into nearly all research disciplines including catalysis.

Carbon is well known in catalysis for two aspects: "coking" that leads to the deactivation of catalysts and as a support for novel metals. The later aspect has begun to be intensively studied because carbon has some advantages over silica and alumina. In electrochemistry and electrocatalysis, carbon as the support is unique, and it cannot be replaced by other materials. Carbon has the property that its surface can be tuned to be basic or acidic by functionlization, and its bulk structure can be tuned by graphitizaton at various temperatures. Another important aspect of carbon materials is that they can accommodate micro-, meso-, and macropores, which can provide either very high specific surface areas or fast mass transport. Recently, all the allotropes of carbon have been studied as a metal-free catalyst that is a better alternative to traditional metal-containing catalysts. This is a relatively young research area that is not yet in the mainstream of catalysis, but it is very promising as the papers in this special issue will show.

The requirement for the sustainable development of our society raises new challenges for catalysis to develop new catalysts and new processes that are energy- and resource-saving and cost-effective. This opens new chances for carbon materials as support and catalyst in catalysis. In addition to nano- structured carbons, hybrid carbons, which are carbon nano-architectures that consist of several of the above nanocarbon building blocks, are attracting more research interests for complicated processes or reactions. Another trend in "Carbon in Catalysis" is the use of carbon for many energy-related processes or reactions, including $\mathrm{CO}_{2}$ utilization and biomass conversion.

This special issue is an update of the state-of-art of "Carbon in Catalysis" that covers all aspects, including production, preparation, functionalization, characterization, and application, of carbon as support and catalyst. The high quality of all the papers written by international well-known research groups in this highly exciting research area will render this special issue a good reference for future research activity.

I express my sincere thanks to all the authors who are my best friends and colleagues.

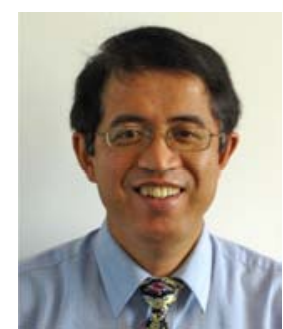

Dr. Dangsheng Su (Guest Editor) Shenyang National Laboratory for Materials Science Institute of Metal Research

Chinese Academy of Sciences Shenyang 110006, Liaoning, China

E-mail address: dssu@imr.ac.cn 